\title{
EROSIÓN HÍDRICA EN LA CUENCA ALTA DEL RÍO MOCHE
}

\section{WATER EROSION IN THE HIGH BASIN OF THE MOCHE RIVER}

\author{
Pablo Huerta Fernández y Oscar Loli Figueroa ${ }^{2}$
}

\begin{abstract}
Resumen
El objetivo del trabajo fue estudiar la pérdida de suelo y la incidencia de las precipitaciones pluviales como factor en la erosión hídrica. Se cuantificaron la escorrentía superficial y la pérdida de suelo bajo condiciones de uso del suelo en las microcuencas de Huangamarca y Pollo de la cuenca alta del río Moche. Para este efecto, se determinaron los caudales de los ríos y se utilizó el registro de lluvias de un período de 24 años (1988-2011) de la Estación Meteorológica del SENAMHI. Este dato permitió estimar el poder erosivo de las precipitaciones como factor determinante de la erosión en términos de degradación específica en $\mathrm{t} / \mathrm{ha} / \mathrm{año}$ mediante el método de Fournier, basado en la función fundamental del coeficiente $\mathrm{p}^{2} / \mathrm{P}$, siendo $\mathrm{p}$ la precipitación del mes más lluvioso y $\mathrm{P}$ la precipitación total anual. Se registró pérdidas de suelo por erosión hídrica, en Huangamarca y Pollo, en el orden de 7.29 y 33.40 t/ha/año, respectivamente. Estas pérdidas por erosión son consideradas valores altos, comparados con el rango permisible por la FAO (0.4-1.8 t/ha/año), que afectan los agroecosistemas.
\end{abstract}

Palabras clave: erosión hídrica, pérdida de suelo, cuenca.

\begin{abstract}
The objective of this work was to study soil loss and incidence of pluvial precipitation as a factor in erosion. Surface runoff and soil loss under the terms of land use in the Huangamarca and Pollo watersheds of the river Moche were quantified. For this purpose, the rivers' discharge using rainfall records over a period of 24 years (1988-2011) as registered by the SENAMHI weather station were determined. Data allowed the estimation of the erosive power of rainfall as a determinant of erosion in terms of specific degradation in $t /$ ha/year. The Fournier method, based on the fundamental role of the $\mathrm{p}^{2} / \mathrm{P}$ ratio, where $p$ is the precipitation of the wettest month and $P$ is the annual total precipitation, was used. Soil losses by water erosion were obtained in the order of 7.29 and 33.40 t/ha at Huangamarca and Pollo basins, respectively. These losses by erosion are considered as high values, compared to the permissible range by FAO $(0,4-1,8 \mathrm{t} / \mathrm{ha} / \mathrm{yr})$, that affect agro-ecosystems.
\end{abstract}

Key words: erosion, soil loss, basin.

\section{Introducción.}

La pérdida de suelo por efectos de la erosión constituye uno de los principales problemas a nivel mundial; la erosión reduce la capacidad del suelo de retener el agua (FAO, 1980). Nuestro país, es muy heterogéneo en las características de los suelos en su relación con la erosión (erodabilidad) y con la incidencia de la precipitación, la misma que fluctúa aproximadamente de 10 a $3000 \mathrm{~mm}$ por año, con una distribución anual irregular, siendo menor en el periodo de abril a noviembre y mayor de diciembre a marzo. Esta situación afecta a su duración como a su intensidad (erosividad). El agua proveniente de la precipitación, no infiltra en su totalidad por lo que una parte se queda en la superficie originando el agua de escorrentía, que al desplazarse sobre la superficie del suelo origina la erosión laminar, en surcos y posteriormente en cárcavas.
La erosividad de la lluvia, en términos generales es la capacidad o poder de la lluvia para causar la pérdida de suelo (Nearing et al., 2004). Está más generalizado el concepto en términos del factor $\mathrm{R}$ (erosividad de la lluvia) en la USLE (Universal Soil Loss Equation) (Wischmeier \& Smith, 1978).

La erosión así planteada afecta la producción de los cultivos, causando la degradación de tierras que es un fenómeno de gran interés mundial, al igual que otros como el cambio climático, la pérdida de biodiversidad, la escasez de agua y las sequías (Pulido \& Bocco, 2011).

La degradación de los suelos debido a fenómenos hidrológicos disminuye sus cualidades como recurso natural y su productividad en el agroecosistema. La escorrentía permite la pérdida de elementos presentes en el suelo, principalmente de aquellos que se mueven por flujo de masas (Lasanta \& García, 1999). 
Las gotas de lluvia poseen energía cinética producto a su masa y velocidad de caída, las cuales al caer sobre el suelo, inciden en las propiedades del suelo como la estructura y compactación, que se ven seriamente afectadas, formándose el suelo superficial, estas redundan en una menor infiltración y por lo tanto en mayor cantidad de agua de escorrentía (Sánchez \& Cuadrado, 1986 ); pueden ser medibles estas variables de acuerdo con las precipitaciones, pendiente y exposición del suelo (Lasanta \& García, 1999).

La erosión hídrica, debida a las precipitaciones, es ocasionada por la dispersión y transporte del suelo a causa del impacto de las gotas de lluvia conjuntamente con escurrimiento superficial del agua. Esto depende principalmente de factores y de sus interrelaciones, como a) cantidad e intensidad de las precipitaciones; b) propiedades intrínsecas de los suelos, que según los estudios presentan una inestabilidad estructural, con predominancia de estructura granular y del grupo textural liviano con predominancia de la clase franco arena, configurando una propiedad de permeabilidad, que los hacen vulnerables; y c) inclinación y área del terreno, vegetación y aplicación de medidas preventivas para la conservación del suelo.

Las características ecológicas y socioeconómicas, en un territorio, son determinantes en los estudios de erosión, tanto en lo relacionado con el factor erodabilidad como erosividad (Ehlers, 1999).

Para este efecto se debe considerar que la mayor parte de los suelos estudiados y dedicados a cultivos transitorios en la provincia de Otuzco se hallan afectados por importantes procesos de degradación por erosión hídrica, influenciado por la pendiente e inadecuado manejo de los suelos.

En este sentido, el objetivo del presente trabajo es determinar la pérdida de suelos, efectuando estudios de campo en las microcuencas seleccionadas. Para lograr este objetivo se ha obtenido información meteorológica del Servicio Nacional de Meteorología e Hidrología (SENAMHI) y también se han realizado estudios de escorrentía como factor de la erosión hídrica con base a los datos obtenidos de los pluviómetros instalados en las dos microcuencas estudiadas.

\section{Materiales y métodos.}

El área de trabajo se ubicó en las microcuencas Huangamarca (coordenadas UTM Este 781899 Norte 9130090) a 3440 msnm y Pollo (coordenadas UTM Este 768688 Norte 9125564) a 2625 msnm, ubicadas en la parte alta de la cuenca del río Moche, (Mapas en anexos 1 y 2).

Se procedió a un reconocimiento general del área apoyada en el mapa cartográfico y trabajo de campo para obtener datos geomorfológicos, edáficos, hidrológicos y de uso de los suelos en las microcuencas seleccionadas Huangamarca y Pollo pertenecientes a la subcuenca Otuzco de la provincia del mismo nombre, territorio de la cuenca hidrográfica del río Moche.

Se efectuó la selección en razón a que las citadas microcuencas, son zonas vulnerables, fundamentalmente por presentar pendientes y escasa cobertura vegetal; y por significar las despensas más cercanas para el abastecimiento de productos agropecuarios a la localidad de Otuzco y a la ciudad de Trujillo (La Libertad). Por su importancia ecológica, económica y social, se definieron como espacios de estudio con base a la información cartográfica del Instituto Geográfico Nacional (IGN), que permitieron conocer características geomorfológicas y edafológicas.

Se registraron caudales de agua, por el método velocidad/superficie ${ }^{1}$, resultantes de la escorrentía en el período comprendido de octubre 2005 a mayo 2006; registros realizados en cuatro momentos en los ríos de la cuenca, a los efectos de determinar el índice de escorrentía y el coeficiente de escorrentía. Los registros se realizaron en función a periodos de lluvias, al inicio de las lluvias (octubre-noviembre); segundo periodo (diciembre-enero); tercer periodo (febrero-marzo) de mayor precipitación; y cuarto periodo (abril-mayo) de baja precipitación; periodos estacionales que permitieron registrar el caudal promedio anual.

Llamamos escorrentía a la lámina de agua que circula en una cuenca de drenaje, es decir la altura en milímetros de agua de lluvia escurrida y extendida uniformemente. Normalmente se considera como la precipitación menos la evapotranspiración real.

Si conocemos el caudal relativo (módulo relativo) del río podemos obtener el índice de escorrentía multiplicándolo por un valor constante: 31.557 que es el factor de conversión para expresar el resultado final en milímetros.

$$
\begin{aligned}
& \text { Índice de escorrentía } \\
& \mathrm{Ie}=\mathrm{Mr}\left(1 / \mathrm{seg} / \mathrm{km}^{2}\right) \cdot 31.557 \\
& \mathrm{Ie}=\text { Índice de escorrentía }(\mathrm{mm}) \\
& \mathrm{Mr}=\text { Caudal relativo (modulo relativo expresado } \\
& \text { en } \left.1 / \mathrm{seg} / \mathrm{km}^{2}\right)
\end{aligned}
$$

El caudal relativo es la relación entre el caudal medio anual y los $\mathrm{km}^{2}$ de cuenca de río.

$$
\text { Coeficiente de escorrentía }
$$

El coeficiente de escorrentía es la relación entre el índice de escorrentía y la precipitación anual. Indica qué porcentaje de la precipitación media anual circula; relaciones que se indican a continuación:

$\mathrm{Ce}=\mathrm{Ie} / \mathrm{Pmm}$

Ce expresado en tanto por ciento:

$\mathrm{Ce}=(\mathrm{Ie} / \mathrm{Pmm}) \cdot 100$

$\mathrm{Ce}=$ Coeficiente de escorrentía

$\mathrm{Ie}=$ Índice de escorrentía $(\mathrm{mm})$

\footnotetext{
${ }^{1}$ El método de velocidad/superficie consiste en la medición de la velocidad media de la corriente y del área de sección transversal por el cual fluye el agua.
} 
Pmm $=$ Precipitaciones anuales en milímetros

Los registros de precipitaciones, para los estudios de escorrentía fueron obtenidos de los pluviómetros instalados en las cabeceras de las microcuencas Huangamarca y Pollo (Tabla 1).

Los registros de precipitación (Tabla 2 del anexo) se realizaron de un período de 24 años (1988 2011) de la Estación Meteorológica de Salpo, del Servicio Nacional de Meteorología e Hidrología (SENAMHI), ubicado a $3.7 \mathrm{~km}$ del ámbito de estudio. Data de largo periodo, representativo para las estimaciones correspondientes.

La estimación del poder erosivo de las precipitaciones, expresada como degradación específica en t/ha/año se efectuó por el método empírico de Fournier (1960) basado en la función fundamental del coeficiente $\mathrm{p}^{2} / \mathrm{P}$. Siendo $\mathrm{p}$ la precipitación del mes más lluvioso, y $\mathrm{P}$ la precipitación total anual, lo que permite hallar los valores de erosión potencial. Este índice, ha sido ampliamente usado como descripción de la agresividad de las precipitaciones, aún más el índice modificado de Fournier (Arnoldus, 1980). Según Morgan (2005), este índice fue usado por Low (1967) para el estudio de la variación regional del riesgo de erosión en Perú.

El procedimiento propuesto por F. Fournier (1960) trata de evaluar la degradación específica de una cuenca, con base en sólo dos factores que intervienen en el fenómeno de la erosión: el clima, como precipitación y temperatura, y el relieve.

Para ello establece como parámetros representativos de ambos factores los siguientes:

- El índice de agresividad del clima, definido por la relación $\mathrm{p}^{2} / \mathrm{P}$. Esta evaluación del factor climático se complementa con la consideración del tipo de clima de la cuenca.

- Para el relieve se emplea el coeficiente orográfico: h.tg $\alpha$, siendo $h$, la altura media del relieve de la cuenca en $\mathrm{m}$. y $\operatorname{tg} \alpha$ el coeficiente de masividad de Martone: $\operatorname{tg} \alpha=\mathrm{h} / \mathrm{S}$; donde $\mathrm{S}$ es la proyección horizontal de la superficie de la cuenca en $\mathrm{km}^{2}$. Este coeficiente caracteriza eficazmente el relieve de 2 cuencas distintas. Siendo mayor de 6 el relieve es accidentado y si es menor de 6 , es poco accidentado (Mármol, 2008).

El estudio de la correlación lineal establecida entre el valor de $\mathrm{X}$, degradación específica de la cuenca expresada en $\mathrm{t} / \mathrm{ha}$.año o $\mathrm{t} / \mathrm{km}^{2}$.año, y el valor de $\mathrm{Y}$, índice de agresividad del clima en $\mathrm{mm}$, indica un alto grado de confianza, representándose la dependencia entre ambas variables mediante cuatro rectas de regresión, según los valores representativos de los otros parámetros utilizados (Robredo, 1994) de la forma siguiente:

Para h.tg $\alpha<6$; - con $\mathrm{p}^{2} / \mathrm{P}<20: X=6.14 . Y-49.78$
Para h.tg $\alpha>6$ :

- con clima no semiárido: $\quad X=52.49 . Y-513.21$

- con clima semiárido $\quad: \quad X=91.78 . Y-737.62$

Normalmente las rectas de regresión de F. Fournier no deben utilizarse más que para grandes cuencas, superiores a $2000 \mathrm{~km}^{2}$ (Robredo, 1994).

Los procesos de erosión hídrica son complejos, están involucrados los cambios en las cantidades e intensidades de las lluvias, número de días de precipitación, tasa de lluvia, producción de biomasa de plantas, proporciones de descomposición de residuos de plantas, actividad microbiana en el suelo, tasas de evapotranspiración y los cambios necesarios en el uso de la tierra para adaptación al nuevo régimen climático (Nearing et al., 2004).

En los procesos de erosión de suelos, hay tolerancia a la pérdida de suelo, que se define como la cantidad de tierra que, expresada en tonelada por unidad de superficie y año, puede perder un perfil edáfico manteniendo su nivel de productividad actual durante un largo periodo de años (FAO, 1967). Refleja, pues, la máxima pérdida de suelo admisible con un grado de conservación tal que mantenga una producción económica, con los medios técnicos disponibles.

\section{Resultados y discusión.}

\section{Escurrimiento por microcuencas.}

Los datos de caudales y precipitaciones pluviales registrados en las microcuencas estudiadas, permitieron estimar los índices y coeficientes de escorrentía correspondientes (Tabla 1).

\begin{tabular}{lrr}
\multicolumn{3}{c}{ Tabla 1. Estimaciones hidrológicas por microcuenca. } \\
\hline \multirow{2}{*}{ Estimaciones Hidrológicas } & \multicolumn{2}{c}{ Microcuencas } \\
& Huangamarca & \multicolumn{1}{c}{ Pollo } \\
\hline Índice de escorrentía (Ie)* & $1044.85 \mathrm{~mm}$ & $576.54 \mathrm{~mm}$ \\
Precipitaciones anuales & $1200 \mathrm{~mm}$ & $652 \mathrm{~mm}$ \\
$($ Pmm) & $87 \%$ & $92 \%$ \\
$\begin{array}{l}\text { Coeficiente de escorrentía } \\
(\mathrm{Ce}) \mathrm{Ce}=\mathrm{Ie} / \mathrm{Pmm}\end{array}$ & & \\
\hline
\end{tabular}

(*) $\mathrm{Ie}=\mathrm{Mr}\left(1 / \mathrm{seg} / \mathrm{km}^{2}\right) \cdot 31.557$

Caudal Relativo (Mr) en Huangamarca $=4290$ 1/seg

Caudal Relativo (Mr) en Pollo $=7201 / \mathrm{seg}$

Superficie de Huangamarca $=129.56 \mathrm{~km}^{2}$

Superficie de Pollo $=38.06 \mathrm{~km}^{2}$

Como se observa en la Tabla 1, la microcuenca Huangamarca presenta un mayor índice de escorrentía que la microcuenca Pollo. Estos resultados pueden ser explicados si consideramos que las precipitaciones son mayores en Huangamarca, lo que permite un mayor índice de escorrentía; pero por sus características geomorfológicas de menor pendiente, colinas poco prominentes en el área superficial con cobertura vegetal, su coeficiente de escorrentía es menor. 
En la Figura 1, se puede ver el perfil transversal B2-B3, en la parte media de la microcuenca Huangamarca, cuya línea longitudinal cubre entre las cotas 3299 y 3885, este trayecto presenta zonas colinosas que intercalan con relieves de ondulaciones suaves. En estos ámbitos, en algunas áreas, las tierras son trabajadas con el sistema de regadío, con aguas provenientes de las quebradas, que son abundantes en ésta microcuenca; predomina el cultivo de papa (Solanum tuberosum L.), y en menores extensiones se cultivan cereales representados por trigo (Triticuma estivum) y cebada (Hordeum vulgare) y menestras como arveja (Pisum sativum) y haba (Vicia faba); en las que las prácticas agrícolas no son adecuadas.

Las características geomorfológicas descritas, zona de menores pendientes; la presencia de la red hídrica y las infraestructuras viales existentes, configuran un paisaje de sierra favorable para la agricultura; es decir, la microcuenca Huangamarca por el relieve que presenta y la cobertura forestal, su territorio es menos vulnerable, como mencionan Mingoti \& Vettorazzi (2011) el efecto de la cobertura forestal en la reducción de la pérdida de suelo por año, varía de acuerdo al promedio de la pendiente de la microcuenca; de toda forma, la cobertura vegetal en general protege la erosión del suelo.

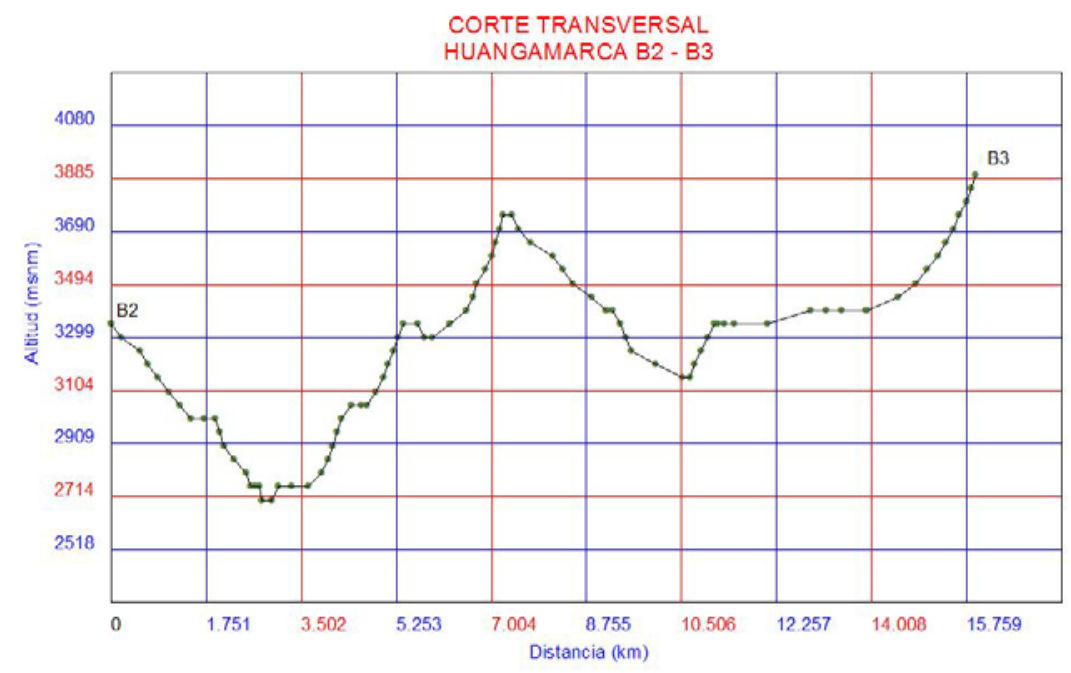

Figura 1. Perfil transversal de la Microcuenca Huangamarca.
En el perfil A2-A3 (Figura 2), que corresponde a la parte media de la microcuenca Pollo, en general se observa un territorio con predominancia de fuertes pendientes, configurando un ecosistema frágil en esta zona media de la microcuenca; siendo la escorrentía mayor en relación a las zonas alta y baja. Como se observa el perfil tiene la forma de "V", formando el río Pollo.

En estas condiciones geomorfológicas poco favorables y fisiografía del medio, limitan el uso de la tierra en el territorio de Pollo (Mapa anexo 3), resulta ser la agricultura una actividad de subsistencia con predominancia de cultivos anuales representados por los cereales, incluido el maíz (Zea maíz); existiendo mayores riesgos por el uso inadecuado de las tierras y el efecto de las condiciones climáticas, fundamentalmente por las precipitaciones pluviales que originan erosión hídrica.

Erosividad de la lluvia

Al efectuar las estimaciones del poder erosivo de las precipitaciones,

expresadas como degradación específica (t/ha/año) mediante el método de Fournier, se han encontrado resultados de pérdida de suelo en la microcuenca de Pollo en una proporción mayor como potencial erosivo 
respecto a la microcuenca de Huangamarca, principalmente por los efectos presentados en la parte alta de las cuencas, cuyos cálculos se pueden observar en la Tabla 3.

Ello lo podemos explicar si consideramos las características geomorfológicas, presencia de colinas en las superficies estudiadas, pendientes, fundamentalmente, de cada una de las microcuencas y la altitud a la cual se presentan; pues son las partes altas las que presentan mayores pendientes, siendo las pertenecientes a la microcuenca de Pollo, las de pendientes más pronunciadas (Figura 2).

En la microcuenca Pollo, no obstante las escasas lluvias presentadas $(484 \mathrm{~mm})$, se registran pérdidas de suelo por escorrentía, resultando un estimado de 33.40 t/ha/año. (Tabla 3), que representa el $78 \%$ más que en Huangamarca, que le corresponde 7.29 t/ha/año, esta vulnerabilidad es función de las condiciones topográficas presentadas y probablemente esté asociada al manejo y uso inadecuado de la tierra. Las fuertes pendientes que hay en Pollo, llegan hasta el 35 $\%$ (fuertes $\mathrm{y}$ escarpadas) en comparación a Huangamarca que presenta geoformas de relieves de menores pendientes que en su mayoría oscilan entre 10 y $15 \%$ (suaves y moderadas). de pérdida de suelo son similares a los reportados en este trabajo, siendo los acumulados de lluvia de Chiapas mayores.

Según los estudios realizados por Alegre (1979) y La Torre (1985), en parcelas de escorrentía determinaron pérdidas de suelos hasta 16 y $70.4 \mathrm{t} / \mathrm{ha}$, respectivamente; en escenarios de selva alta (San Ramón - Chanchamayo) bajo las condiciones experimentales, las precipitaciones pluviales oscilaron entre 1400 y $2000 \mathrm{~mm}$ distribuidos en los meses lluviosos de diciembre a abril. Estos registros de pérdidas de suelos por efecto de la erosión hídrica son buenos referentes y similares a las pérdidas de suelo que encontramos en las montañas de la sierra (microcuencas de Huangamarca y Pollo), como se informa en el presente trabajo, importantes para la orientación sobre las medidas de manejo y conservación de los suelos.

Así mismo, en los trabajos de Alegre et al. (1990) realizados en un período de 4 años en la localidad de Santa Ana (Huancayo-Junín), determinaron pérdidas de suelos de 12.9 hasta 47.2 t/ha, en cuyo rango se encuentra la pérdida de suelo determinada, en el presente estudio, para la microcuenca Pollo (33.4 t/ha/año).

Tabla 3. Degradación específica o erosión potencial en las microcuencas de Huangamarcay Pollo.

\begin{tabular}{|c|c|c|c|c|c|c|c|}
\hline \multirow{2}{*}{ Microcuenca } & \multirow{2}{*}{$\begin{array}{l}\text { Superficie } \\
\quad\left(\mathrm{km}^{2}\right)\end{array}$} & \multirow{2}{*}{$\begin{array}{c}\text { Coef. } \\
\text { Orogr. } \\
(h \times \operatorname{tg} \alpha)\end{array}$} & \multirow{2}{*}{$\begin{array}{c}\text { Precipitación } \\
\text { mensual } \\
\text { Promedio } \\
\text { Máximo (p) }\end{array}$} & \multirow{2}{*}{$\begin{array}{c}\text { Precipitacion } \\
\text { Total } \\
\text { promedio } \\
\text { anual (p) }\end{array}$} & \multirow{2}{*}{$\begin{array}{l}\text { Índice de } \\
\text { fournier } \\
\left(y=p^{2} / p\right)\end{array}$} & \multicolumn{2}{|c|}{$\begin{array}{c}\text { Degradación especifica de las } \\
\text { microcuencas (t/ha/año) }\end{array}$} \\
\hline & & & & & & $X=27,12 . Y-475,4$ & $X=91,78 . Y-737,6$ \\
\hline Huangamar & 129.71 & & 176.6 & 702.05 & & 7.29 & \\
\hline Pollo & 38.06 & 13.71 & 176.6 & 702.05 & 44.42 & & 33.40 \\
\hline
\end{tabular}

Estas cantidades de pérdida de suelo, como potencial de erosión, son altas según la FAO (1980), considerando como riesgo de erosión, en el rango permisible o de tolerancia (entre 0.4 y $1.8 \mathrm{t} / \mathrm{ha} / \mathrm{año}$ ).

Siguiendo este mismo proceso de estimación del poder erosivo de las precipitaciones pluviales, Chamochumbi (1987) en su trabajo desarrollado en la cuenca Lurín (Lima, Perú) estimó el potencial de erosión por el método de F. Fournier, determinando la degradación específica de $30.86 \mathrm{t} / \mathrm{ha} / \mathrm{año}$ como valor mínimo a $64.01 \mathrm{t} / \mathrm{ha} / \mathrm{año}$ como valor máximo, considerados altos y que están por encima de los límites tolerables para las zonas de sierra, coincidente con los datos obtenidos en el presente estudio, que también están por encima de los límites de tolerancia de pérdidas de suelo.

Cabe anotar que, en estudios similares de erosión hídrica, realizado por Santacruz (2011) en la cuenca del río Cahoacán (Chiapas-México) se estimó pérdidas de suelos de 16.27 a 20.02 t/ha/año, presentándose un valor mínimo de $13.31 \mathrm{t} / \mathrm{ha} / \mathrm{año}$; habiéndose registrado precipitaciones entre 1176 y $2000 \mathrm{~mm}$. Estos valores de pérdida de suelo se obtuvo con un rango de variación pequeño en comparación con el rango de variación de las precipitaciones; además, estos valores
Frente a estas situaciones de erosión y riesgos de degradación de los suelos, el uso de la labranza de conservación en áreas agrícolas de una cuenca, reduce las pérdidas de suelo a menos de $3 \mathrm{t} / \mathrm{ha}$ /año (Espinoza et al., 2009).

En este análisis de riesgo de degradación de los suelos, sin perspectivas de sustentabilidad, los ecosistemas van presentando fragilidad en la medida que van perdiendo sus características naturales por los efectos de las condiciones climáticas y también por los procesos antrópicos como signo de sobrevivencia de los pequeños agricultores (Altieri, 1999); debiendo tomarse las medidas prospectivas para evitar el deterioro del medio ambiente.

\section{Conclusiones.}

Las pérdidas de suelo para las microcuencas Huangamarca y Pollo, fueron del orden de 7.29 a $33.40 \mathrm{t} / \mathrm{h} / \mathrm{año}$, respectivamente. Estos valores de las pérdidas de suelo son elevados, considerando los límites de tolerancia propuestos por la FAO (1980), los cuales afectan de manera sistemática a agroecosistemas frágiles. 
La erosión hídrica es mayor en la microcuenca Pollo, por tanto los suelos se caracterizan por presentar una alta erodabilidad. Lo cual incrementa la erosión potencial por las condiciones geomorfológicas poco favorables, fisiografía del medio, escasa cobertura vegetal y el efecto de las precipitaciones pluviales. El factor geomorfológico representado por las pendientes que llegan hasta $15 \%, 35 \%$ en las microcuencas Huangamarca y Pollo, respectivamente, influyen en la erosión de los suelos.

\section{Agradecimientos.}

Hacemos reconocimiento al Programa Nacional de Manejo de Cuencas Hidrográficas y Conservación de Suelos, ahora AGRORURAL, Oficina de Otuzco del Ministerio de Agricultura y Riego.

\section{Literatura citada.}

Alegre J.C. 1979. Medida de la erosión hídrica de un entisol de la selva alta (San Ramón-Chanchamayo) bajo diferentes sistemas de cultivo. Universidad Nacional Agraria La Molina. Tesis para optar el grado de Magister Scientiae. Lima-Perú.

Alegre J.C., Felipe-Morales C. \& La Torre B. 1990. Soil erosion studies in Peru. Journal of soil and water conservation. p. 417 - 418.

Altieri M.A. 1999. "El rol ecológico de la biodiversidad en agro ecosistemas". Universidad de California. Berkeley - CLADES. p. 8

Arnoldus H.M.J. 1980. An approximation of the rainfall factor in the Universal SoilLoss Equation. In M. De Boodt and D. Gabriels. (eds.). Assessment of soil erosion. John Wiley and Sons, Chichester, Gran Bretaña. p. 127-132.

Chamochumbi W. M. 1987. Inventario, evaluación y uso de andenes en la cuenca del río Lurín. Tesis para optar el título de Ingeniero Agrónomo. Universidad Nacional Agraria La Molina. Lima-Perú.

Ehlers E. 1999. Agricultura sustentável. 2a . ed. Livraria e Editora Agropecuária Ltda. Guíaba - Brasil.

Espinoza M., Mendoza M.E. \& Medina L. E. 2009. Escenarios de erosión bajo diferentes manejos agrícolas en la cuenca de lago Zirahuén, Michoacán, México. Investigaciones geográficas, Bol. 68.

FAO. 1967. La Erosión del Suelo por el Agua: Algunas medidas para combatirlas en las tierras de cultivo. Colección FAO: Fomento de Tierras y Aguas $\mathrm{N}^{\circ} 7$.
FAO, Roma. (También FAO: Cuadernos de fomento agropecuario $\left.\mathrm{N}^{\circ} 81\right)$.

FAO. 1980. Metodología provisional para la evaluación de la degradación de los suelos. Roma.

Fournier F. 1960. Climat et erosion. Ed. Presses Universitaires de France. París.

Lasanta T. \& García J. M. 1999. Exportación de solutos desde diferentes usos del suelo. Estudio experimental en el pirineo central español. Instituto Pirenaico de Ecología, CSIC, Campus de Aula Dei. Zaragoza.

La Torre B. 1985. Efecto de sistemas de cultivos sobre la escorrentía, erosión y pérdida de nutrientes en un entisol de la selva alta-CAP José Santos Atahualpa-San RamónChanchamayo. Universidad Nacional Agraria La Molina. Tesis para optar el grado de Magiter Scientiae y el título de Ingeniero Agrónomo. Lima - Perú.

Low F.K. 1967. Estimating potential erosion in developing countries. Journal of Soil and WaterConservation 22: $147-8$.

Mármol L. 2008. Introducción al Manejo de Cuencas Hidrográficas y Corrección de Torrentes. Universidad Nacional de Salta. Salta, Argentina.

Morgan R.P.C. 2005. Soil erosion and conservation. Third edition, Blackwell publishing, Oxford, UK.

Mingoti R. \& Vettorazzi C.A. 2011. Relative reduction in annual soil loss in micro watersheds due to the relief and forest cover. Eng. Agríc. vol.31 no.6. pp. 1202-1211.

Nearing M. A., Pruski F.F. \& O'Neal M. R. 2004. Expected climate change impacts on soil erosion rates: A review. Journal of Soil and Water Conservation, 59(1):43-50

Pulido J. \& G. Bocco. 2011. ¿Cómo se evalúa la degradación de tierras? Panorama global y local. INTERCIENCIA. Rev. Ciencia y Tecnología de América 36: 2.

Robredo J.C. 1994. Estimación de la erosión en la cuenca de recepción. Departamento de Ingeniería Forestal. E.T.S. Ingeniería de Montes. Universidad Politécnica de Madrid, España. pp. 35-36.

Sánchez F. \& Cuadrado S. 1986. El agua y el medio físico del suelo. EUROPA Artes Gráficas, S.A. Salamanca España.

Santacruz G. 2011. Estimación de la erosión hídrica y su relación con el uso de suelo en la cuenca del río Cahoacán, Chiapas, México. Rev. Agua-LAC 3: 1 (4554).

Wishmeyer W.H. \& D.D. Smith. 1978. Predicting rainfall erosion losses: a guide to conservation planning. Washington: USDA Agricultural Handbook. 


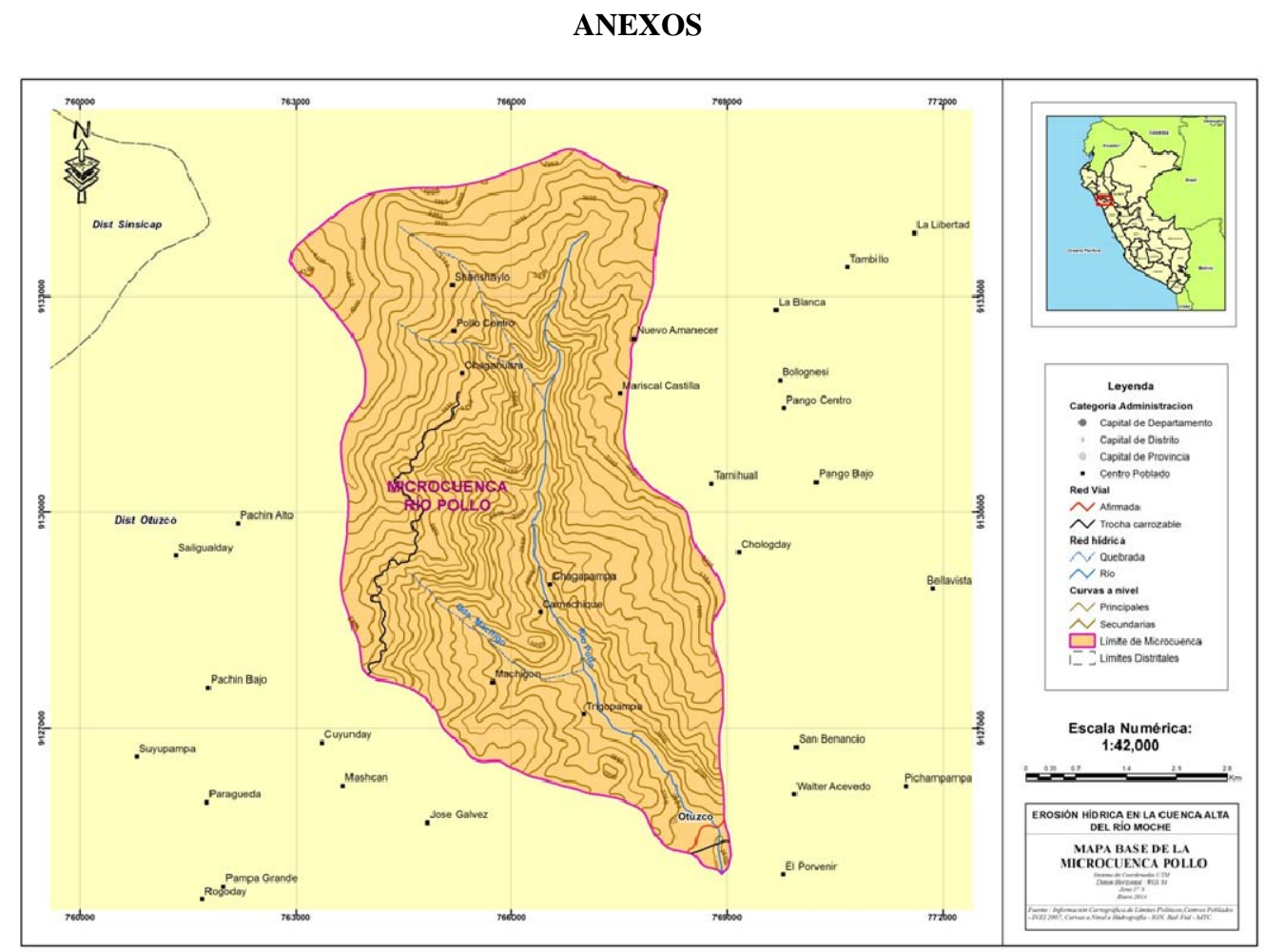

Anexo 1. Mapa base de Microcuenca Pollo

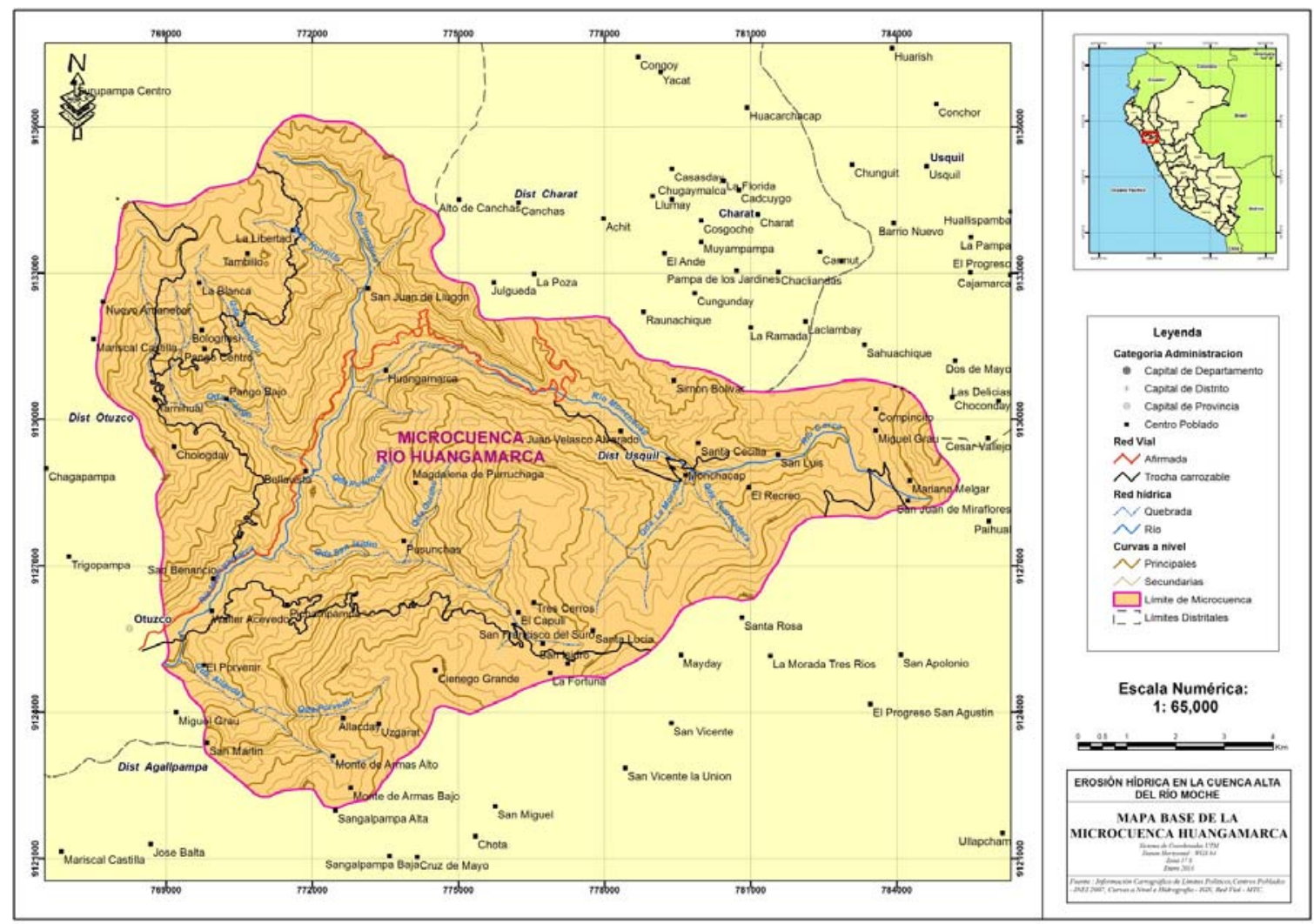

Anexo 2. Mapa base de Microcuenca Huangamarca 


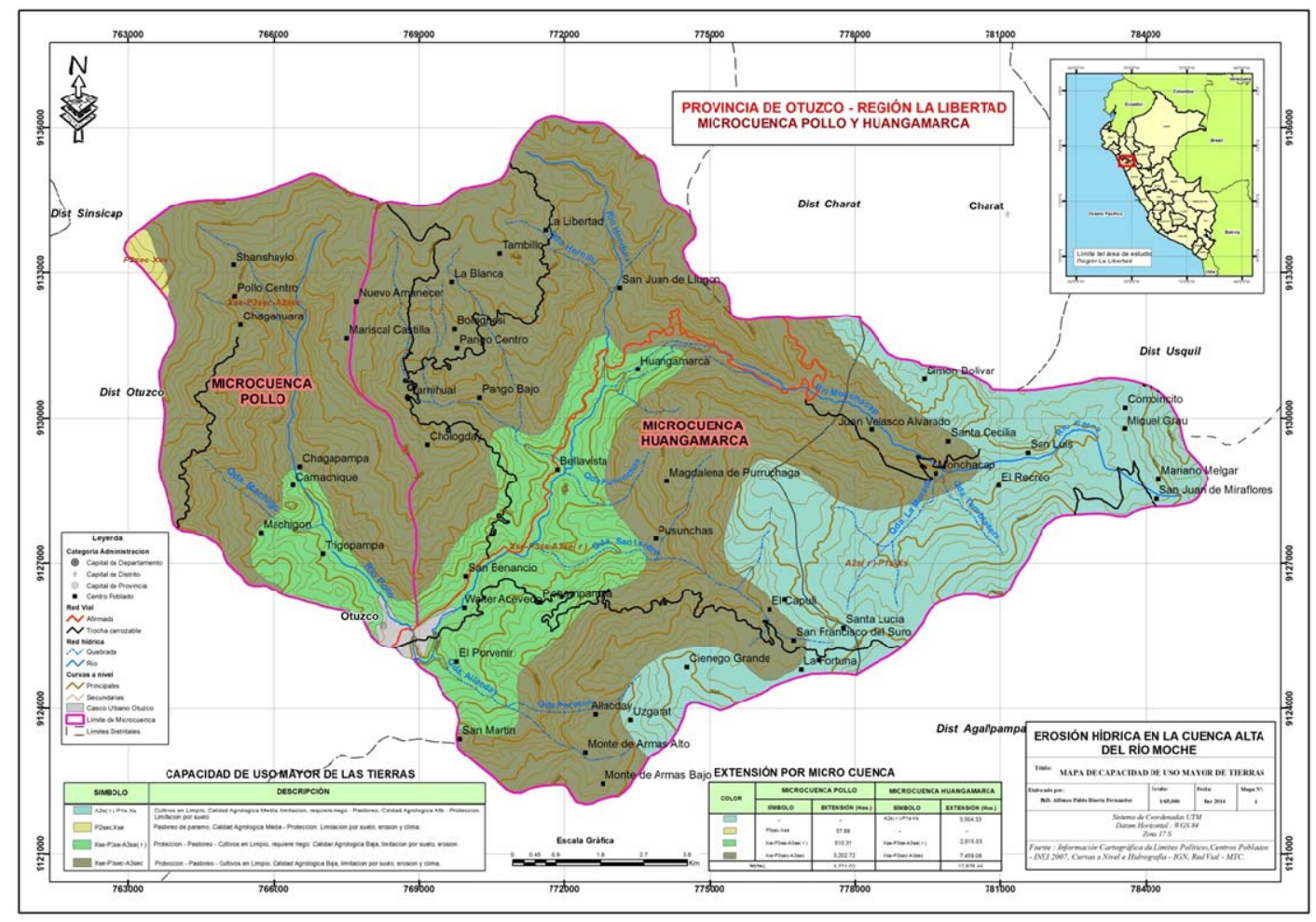

Anexo 3. Mapa de capacidad de uso mayor de tierras

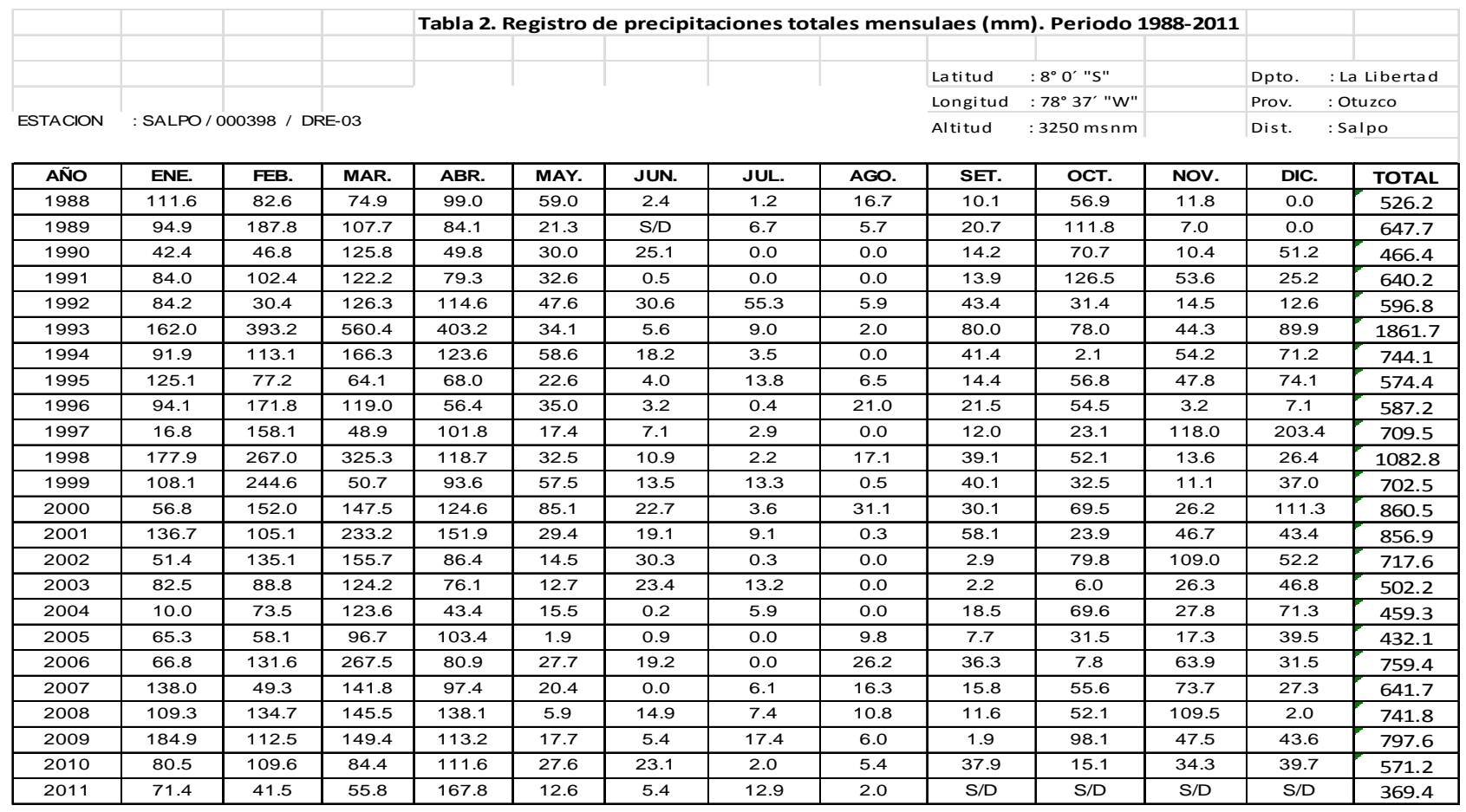

${ }^{1}$ Ing. Agr. Dr. Responsable de Formación de Recursos Humanos en Ciencia y Tecnología-CONCYTEC. Calle Madrid No 116, Dpto. 101, Miraflores-Lima. apablohuerta@hotmail.com

${ }^{2}$ Ing. Agr. Dr. Profesor Principal, UNALM. Av. Javier Prado No 3502, San Borja-Lima. ololi@lamolina.edu.pe 